\title{
PROPOSED PIECEWISE LINEAR APPROXIMATION FOR TACKLING NONLINEAR PROGRAMMING PROBLEMS WITH SEPARABLE OBJECTIVE FUNCTION
}

\author{
OGBONNA, CHUKWUDI J. \\ Department of Statistics, Federal University of \\ Technology, Owerri, Imo State, Nigeria
}

\author{
IHEAGWARA ANDREW I. \\ Director, Program Analyst, State Bureau \\ of Statistics \& Director PRS, Board of \\ Internal Revenue, Owerri, Imo State, Nigeria
}

\begin{abstract}
The study has developed an algorithm that can be employed to tackle optimization problems with nonlinear separable quadratic objective function with linear constraints. The algorithm used two breakpoints. After transforming the nonlinear objective function to linear function, Wolfram Mathematica was employed to get the optimal solution. The two problems solved in this study showed that the proposed algorithm converged faster than solving the original problem directly via Wolfram Mathematica, though with the same optimal solution. In the study, a program code via Wolfram Mathematica for evaluating a nonlinear separable quadratic objective function with multiple linear variables and constraints of different sizes up to 70,000 variables and 35,000 constraints was written using the proposed technique. The study demonstrated the effectiveness of the proposed approach using the written program code as compared to the written program code for the original problem as it converged faster via the time of execution.
\end{abstract}

\section{Keywords - Nonlinear Programming Problem, Separable Quadratic Objective Function, Piecewise Linear Approximation}

\section{INTRODUCTION}

Mathematical optimization or mathematical programming problem is the main problem to be considered in this study. Mathematical programming problem seeks to minimize (or maximize) a function of many variables subject to a set of constraints on these variables.

If the objective and constraints of an optimization problem are given as mathematical functions and functional relations, it is called mathematical programming (Dey and Gupte, 2015).

\author{
OPARA JUDE \\ Department of Statistics, Michael \\ Okpara University of Agriculture, \\ Umudike Umuahia, Abia State, Nigeria \\ IHEKUNA STEPHEN, O. \\ Department of Statistics, Imo State \\ University, Owerri, Imo State, Nigeria
}

Linear Programming (LP, also called linear optimization) is a method to achieve the best outcome (such as maximum profit or lowest cost) in a mathematical model whose requirements are represented by linear relationships (Zhang and Wang, 2005). The Linear Programming Problem can be represented mathematically as;

$$
\left.\begin{array}{c}
\text { Maximize } f(x) \\
\text { Subject to } A(x) \leq b \\
x \geq 0
\end{array}\right\}
$$

The term 'non linear programming' usually refers to the problem in which the objective function in Equation (1a) becomes non-linear, or one or more of the constraint inequalities have non-linear or both (Ashley, 2016). If $f(x)$ is a quadratic function with linear constraints, the problem is called a "quadratic programming problem". For instance, in business, if a firm is seeking to maximize profit or minimize cost, but there are constraints on the amount of resources or monetary funds available, this type of problem comes up (Edirisinghe and Jeong, 2015)

The applications of mathematical optimization problems include also "operational problems such as distribution of goods, production scheduling, and machine sequencing" (Nemhauser and Wolsey; 1999). The common approach of handling with nonlinear programming problems is to try to linearize the problem and then employ one of the numerous available Linear Programming solvers to obtain a good quality approximation (Zhao and de Farias, 2013). 
The method of separable programming was first formulated by Miller (1963). It provides a simple technique for handling arbitrary nonlinear functions of single arguments in otherwise linear programming problems - and can readily be adapted to handle product terms. In a work done by Box (1965), nonlinear programming problem was described as a result of presence of four product terms that arose in his work, for which he described two new computational procedures. Again, there is an aspect of nonlinear programming problem that is known as separable programming problem which is a special case of convex programming which can be expressed as a sum of sub-functions where each sub-function is a function of one variable only (Gupta and Hira, 2011). Hence, in this study, we focus primarily on applying piecewise linear approximation technique to tackle nonlinear programming problems. Particularly, an aspect of nonlinear programming problem whose objective function is separable quadratic function and the constraints are linear.

\section{REVIEW OF RELATED LITERATURES}

Lin et al (2013) researched on "A Review of Piecewise Linearization Methods". Various optimization problems in engineering and management were formulated as nonlinear programming problems. In the last few decades according to the authors, piecewise linearization methods had been widely applied to convert a nonlinear programming problem into a linear programming problem or a mixed-integer convex programming problem for obtaining an approximated global optimal solution. In the transformation process, extra binary variables, continuous variables, and constraints were introduced to reformulate the original problem. These extra variables and constraints mainly determine the solution efficiency of the converted problem. The study provided a review of piecewise linearization methods and analyzed the computational efficiency of various piecewise linearization methods.

Eduardo and Luiz (2015) carried out a study on Models and Algorithms for Optimal Piecewise-Linear Function Approximation. Piecewise-linear functions can approximate nonlinear and unknown functions for which only sample points are available. The study highlighted a range of piecewise-linear models and algorithms to aid engineers to find an approximation that fits best their applications. The models included piecewise-linear functions with a fixed and maximum number of linear segments, lower and upper envelopes, strategies to ensure continuity, and a generalization of these models for stochastic functions whose data points were random variables. Derived from recursive formulations, the algorithms were applied to the approximation of the production function of gas-lifted oil wells.

Having reviewed some of the literatures, this study proposed new technique "piecewise linear approximation" for solving nonlinear programming problems with separable quadratic objective function and linear constraints.

\section{DESCRIPTION OF THE PROPOSED APPROACH}

In describing the proposed approach, consider the problem of the form:

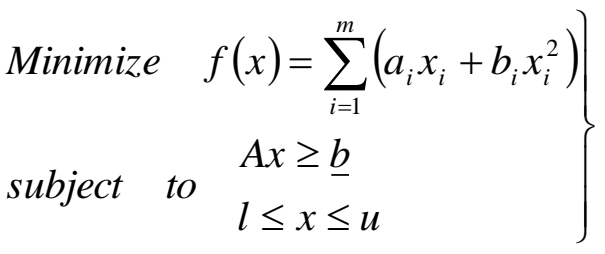

where $\quad x=\left(x_{1}, \ldots, x_{m}\right) \in \mathbb{R}^{\mathrm{m}}$ and $f(x)$ is a nonlinear separable function such that;

$f(x)=f_{1}\left(x_{1}\right)+f_{2}\left(x_{2}\right)+\ldots+f_{m}\left(x_{m}\right)=\sum_{i=1}^{m} f_{i}\left(x_{i}\right)$.

Here we say that a function $f\left(x_{1}, x_{2}, \ldots, x_{m}\right)$ is separable if it can be expressed as the sum of $n$ single-variable-functions $f_{1}\left(x_{1}\right)+f_{2}\left(x_{2}\right)+\ldots+f_{m}\left(x_{m}\right)$ as stated in Equation (2).

The first step of this algorithm is to introduce the Taylor series expansion as:

$e^{x_{i}}=1+x_{i}+\frac{x_{i}^{2}}{2 !}+\frac{x_{i}^{3}}{3 !}+\ldots=\sum_{k=0}^{\infty} \frac{x_{i}^{k}}{k !}$

(3)

Hence Equation (3) is stated as shown in Equation (4)

$e^{x_{i}}=\sum_{k=0}^{\infty} \frac{x_{i}^{k}}{k !}, i=1,2, \ldots, m$

Making $x_{i}^{2}$ the subject of the relation in Equation (3), we get Equation (5)

$x_{i}^{2}=2\left[e^{x_{i}}-1-x_{i}-\sum_{k=3}^{\infty} \frac{x_{i}^{k}}{k !}, i=1,2, . ., m\right] \ldots$

For this algorithm, we shall employ dichotomous breakpoints $(1 / 2 \&$ \& $)$ and evaluate $x_{i}^{2}$ in Equation (5) given $x_{i}=\frac{1}{2}$ and 1 . The values obtained shall be used to form a linear function which approximates the nonlinear objective function. Evaluation of $x_{i}^{2}$ (for $\mathrm{i}=1,2, \ldots, \mathrm{m}$ ) in Equation (5) gave 0.25 and 1 respectively, which then forms $0.25 x_{i 1}+x_{i 2}$ (for $\mathrm{i}=1,2, \ldots, \mathrm{m}$ ), and the result will be substituted into the objective function of Equation (1) to give Equation (6). 


$$
\left.\begin{array}{cl}
\text { Minimize } & f(x)=\sum_{i=1}^{m}\left[a_{i} x_{i}+b_{i}\left(0.25 x_{i 1}+x_{i 2}\right)\right] \\
A x \geq b \\
\text { subject to } \quad l \leq x \leq u, l=0, u=1 \\
& x_{i 1}+x_{i 2}-x_{i}=0, i=1,2, \ldots, m
\end{array}\right\}
$$

The last constraint/s is/are needed to maintain the relationship between the original and new variables.

\section{A. Computer Written Program for Multiple} Variables and Constraints for Nonlinear Problems

Clear[fn,a,x,z,w,h,f,m,n,i,tim];

$\mathrm{n}=\mathrm{q}$; where $\mathrm{q}$ is the number of constraints

$f\left[x_{-}\right]:=\operatorname{Sum}\left[\left(x[2 i-1]^{\wedge} 2\right)+x[2 i]^{\wedge} 2+50 * x[2 i-1]+x[2 i],\{i, n\}\right]$;

$\mathrm{fn}=\operatorname{Sum}\left[\left(\mathrm{x}[2 \mathrm{i}-1]^{\wedge} 2\right)+\mathrm{x}[2 \mathrm{i}]^{\wedge} 2+50 * x[2 \mathrm{i}-1]+\mathrm{x}[2 \mathrm{i}],\{\mathrm{i}, \mathrm{n}\}\right]$;

$\mathrm{a}=$ Variables[fn];

$\mathrm{z}=$ Variables[fn];

$\mathrm{h}=$ Table $[\mathrm{x}[\mathrm{i}]<=1,\{\mathrm{i}, 1,2 \mathrm{n}\}]$;

$\mathrm{m}=$ Table $[\mathrm{x}[2 \mathrm{i}]+\mathrm{x}[2 \mathrm{i}-1]>=1,\{\mathrm{i}, 1, \mathrm{n}\}]$;

$\mathrm{w}=\mathrm{J}$ oin $[\mathrm{m}, \mathrm{h}]$;

tim=Timing[NMinimize $[\{\mathrm{fn}, \mathrm{w}\}, \mathrm{a}]]$

\section{B. Computer Written Program for Multiple Variables and Constraints for Nonlinear Problems for the Proposed Algorithm}

Clear[n,c,f,m,p,h,r,w,k,x,i,z,fmin]

$\mathrm{n}=\mathrm{q}$; where $\mathrm{q}$ is the number of constraints

$\mathrm{c}=2 \mathrm{n}$;

$\mathrm{z}=\operatorname{Sum}[(0.25 * \mathrm{x}[4 \mathrm{i}-3+\mathrm{c}])+\mathrm{x}[4 \mathrm{i}-2+\mathrm{c}]+(0.25 * \mathrm{x}[4 \mathrm{i}-$

$1+\mathrm{c}])+x[4 \mathrm{i}+\mathrm{c}]+50 * x[2 \mathrm{i}-1]+\mathrm{x}[2 \mathrm{i}],\{\mathrm{i}, \mathrm{n}\}]$;

$\mathrm{m}=$ Table $[\mathrm{x}[2 \mathrm{i}-1]+\mathrm{x}[2 \mathrm{i}]>=1,\{\mathrm{i}, 1, \mathrm{n}\}]$;

$\mathrm{p}=$ Table $[\mathrm{x}[2 \mathrm{i}-1+\mathrm{c}]+\mathrm{x}[2 \mathrm{i}+\mathrm{c}]-\mathrm{x}[\mathrm{i}]==0,\{\mathrm{i}, 1, \mathrm{n}\}]$;

$\mathrm{v}=$ Table $[\mathrm{x}[2 \mathrm{i}-1+\mathrm{c}]+\mathrm{x}[2 \mathrm{i}+\mathrm{c}]-\mathrm{x}[\mathrm{i}]==0,\{\mathrm{i}, \mathrm{n}+1,2 \mathrm{n}\}]$;

$\mathrm{h}=$ Table $[\mathrm{x}[\mathrm{i}]<=1,\{\mathrm{i}, 1,2 \mathrm{n}\}]$;

$\mathrm{r}=$ Table $[\mathrm{x}[\mathrm{i}]>=0,\{\mathrm{i}, \mathrm{c}+1, \mathrm{c}+2 \mathrm{c}\}]$;

$\mathrm{w}=\mathrm{Join}[\mathrm{m}, \mathrm{p}, \mathrm{v}, \mathrm{h}, \mathrm{r}]$;

$\mathrm{k}=$ Variables $[\mathrm{z}]$;

$\mathrm{t}=\mathrm{NMinimize}[\{\mathrm{z}, \mathrm{w}\}, \mathrm{k}] / / \mathrm{Timing}$

$\mathrm{f}\left[\mathrm{x}_{-}\right]:=\operatorname{Sum}\left[\left(\mathrm{x}[2 \mathrm{i}-1]^{\wedge} 2\right)+\mathrm{x}[2 \mathrm{i}]^{\wedge} 2+50 * \mathrm{x}[2 \mathrm{i}-1]+\mathrm{x}[2 \mathrm{i}],\{\mathrm{i}, \mathrm{n}\}\right]$;

Table $[\{x[2 i-1]=\{0\}, x[2 i]=\{1\}\},\{i, 1, n\}]$;

fmin $=\mathrm{f}[\mathrm{x}]$;

fmin//Timing

\section{2-dimensional example}

Solve the Nonlinear programming problem (NLPP) using the proposed algorithm.

Min. $f=-4 x_{1}^{2}-3 x_{2}^{2}+6 x_{1}+0.5 x_{2}$

Subject to: $5 x_{1}+x_{2} \geq 3$

$$
0 \leq x_{1} \leq 1
$$

$$
0 \leq x_{2} \leq 1
$$

(Extracted from Ashley, 2016)

\section{Solution}

To make the objective function of the problem to be linear, we employ the objective function of Equation (6) to obtain;

Min. $f=6 x_{1}-4\left(0.25 x_{11}+x_{12}\right)+0.5 x_{2}-$

$3\left(0.25 x_{21}+x_{22}\right)$

or

Min. $f=6 x_{1}-x_{11}-4 x_{12}+0.5 x_{2}-0.75 x_{21}-3 x_{22}$

Hence, using Equation (6), the NLPP becomes LPP as shown in Equation (7)

$$
\begin{aligned}
& \text { Min. } f=6 x_{1}-x_{11}-4 x_{12}+0.5 x_{2}-0.75 x_{21}-3 x_{22} \\
& \text { S.t.: } 5 x_{1}+x_{2} \geq 3 \\
& 0 \leq x_{1} \leq 1 \\
& 0 \leq x_{2} \leq 1 \\
& x_{11}+x_{12}-x_{1}=0 \\
& x_{21}+x_{22}-x_{2}=0
\end{aligned}
$$

Solving this problem in Wolfram Mathematica yields the optimal solution:

$x_{1}=0.4, x_{11}=0, x_{12}=0.4, x_{2}=1, x_{21}=0, x_{22}=1$ with the objective value of

$$
f=-4(0.4)^{2}-3(1)^{2}+6(0.4)+0.5(1)=-0.74
$$

\section{3-dimensional example}

Solve the Nonlinear programming problem (NLPP) using the proposed algorithm.

$$
\text { Min. } f=6 x_{1}+0.5 x_{2}+4 x_{3}-2 x_{1}^{2}-1.5 x_{2}^{2}-1.75 x_{3}^{2}
$$

Subject to: $5 x_{1}+x_{2} \geq 3$

$$
\begin{gathered}
2 x_{3} \geq 1 \\
0 \leq x_{1}, x_{2}, x_{3} \leq 1
\end{gathered}
$$

(Extracted from Ashley, 2016)

\section{Solution}

To make the objective function of the problem to be linear, we employ the objective function of Equation (6) to obtain;

Min. $f=6 x_{1}-2\left(0.25 x_{11}+x_{12}\right)+0.5 x_{2}-$

$1.5\left(0.25 x_{21}+x_{22}\right)+4 x_{3}-1.73\left(0.25 x_{31}+x_{32}\right)$

Min. $f=6 x_{1}-0.5 x_{11}-2 x_{12}+0.5 x_{2}-0.375 x_{21}-$

$1.5 x_{22}+4 x_{3}-0.4375 x_{31}-1.75 x_{32}$ 
Hence, using Equation (6), the NLPP becomes LPP as shown in Equation (8)

$$
\begin{gathered}
\text { Min.f }=6 x_{1}-0.5 x_{11}-2 x_{12}+0.5 x_{2}-0.375 x_{21} \\
-1.5 x_{22}+4 x_{3}-0.4375 x_{31}-1.75 x_{32} \\
\text { S.t. }: 5 x_{1}+x_{2} \geq 3 \\
2 x_{3} \geq 1 \\
0 \leq x_{1} \leq 1 \\
0 \leq x_{2} \leq 1 \\
0 \leq x_{3} \leq 1 \\
x_{11}+x_{12}-x_{1}=0 \\
x_{21}+x_{22}-x_{2}=0 \\
x_{31}+x_{32}-x_{3}=0
\end{gathered}
$$

Solving this problem in Wolfram Mathematica yields the optimal solution:

$$
\begin{aligned}
& x_{1}=0.4, x_{11}=0, x_{12}=0.4, x_{2}=1, x_{21}=0, x_{22}=1, \\
& x_{3}=0.5, x_{31}=0, x_{32}=0.5
\end{aligned}
$$

with the objective value of

$$
\begin{aligned}
& f=6(0.4)+0.5(1)+4(0.5)-2(0.4)^{2}- \\
& 1.5(1)^{2}-1.75(0.5)^{2}=2.6425
\end{aligned}
$$

\section{CONCLUSION}

The study has developed an algorithm that can be employed to tackle optimization problems with nonlinear separable quadratic objective function with linear constraints. The algorithms used two breakpoints. After transforming the nonlinear objective function to linear function, Wolfram Mathematica was employed to get the optimal solution. The two problems solved in this study showed that the proposed algorithm converged faster than solving the original problem directly via Wolfram Mathematica, though with the same optimal solution.

Having concluded this research, it is recommended that future researchers should extend this work by obtaining a direct technique of solving a problem of this nature other than transforming to linear first before solving it, as encountered in this study. Again, a program code should be written after proposing the technique.

\section{ACKNOWLEDGEMENT}

Authors appreciate everybody whose materials served as a reference or information necessary to effectively carry out this study.

\section{REFERENCES}

[1] Ashley, C. (2016). Piecewise linear approximation for nonlinear programming problems. An unpublished $\mathrm{PhD}$. Thesis submitted to the department of Statistics and Mathematics, Graduate Faculty of Texas Tech University, (pp. 1-95).

[2] Box, M. J. (1965). "A new method of constrained optimization and a comparison with other methods," The Computer Journal,Vol. 8, (pp. 42).

[3] Dey, S. S. and Gupte, A. (2015). Analysis of MILP techniques for the pooling problem, Operations Research, 62 (2015), (pp. 412-427).

[4] Edirisinghe, C. and Jeong, J.(2015). An efficient global algorithm for a class of indefinite separable quadratic programs, Mathematical Programming, (2015), (pp. 131).

[5] Eduardo, C. and Luiz, F.N. (2015). Models and Algorithms for Optimal Piecewise-Linear Function Approximation. Hindawi Publishing Corporation Mathematical Problems in Engineering Volume 2015, Article ID 876862, (pp. 1-9).

[6] Ekezie, D.D and Opara, J. (2013). The Application of Transportation Algorithm with Volume Discount on Distribution Cost in Port Harcourt Flour Mills. Journal of Emerging Trends in Engineering and Applied Sciences (JETEAS). 4(2), (pp. 258-272).

[7] Gupta, P.K. and Hira, D.S. (2011). Operations Research. S. Chand \& Company Ltd.,7361, Ram Nagar, New Delhi, (pp. 1308).

[8] Lin, M.H., Carlsson, J.G., Ge, D., Shi, J., Tsai, J.F. (2013). A Review of Piecewise Linearization Methods. Hindawi Publishing Corporation Mathematical Problems in Engineering. Volume 2013, Article ID 101376 (pp. 1244-1251).

[9] Miller, C. E. (1963). "The Simplex Method for Local Separable Programming" from Recent Advances in Mathematical Programming, (pp. 89).

[10] Nemhauser, G. and Wolsey, L. (1999). Integer and Combinatorial Optimization, John Wiley \& Sons, Inc., New York, (pp.439-452).

[11] Zhang, H. and Wang, S. (2005). Global optimization of separable objective functions on convex polyhedra via piecewise-linear approximation. Journal of Computational and Applied Mathematics 197 (2006), (pp. $212-217$ ).

[12] Zhao, M. and de Farias, I. R. (2013). The piecewise linear optimization polytope: new inequalities and intersection with semi-continuous constraints, Mathematical Programming, 141 (2013), (pp. 217-255). 